\title{
Brain Cancer Detection
}

\author{
Akshay Saste $^{1}$, Akash Poduval ${ }^{2}$, Rajeshwari Bathe ${ }^{3}$, Shubham Sethiya ${ }^{4}$, Prof. Pavan Kulkarni ${ }^{5}$ \\ Student, Comp Dept, KJEI'S Trinity College of Engineering and Research, Pune, India ${ }^{1,2,3,4}$ \\ Guide, Comp Dept, KJEI'S Trinity College of Engineering and Research, Pune, India ${ }^{5}$
}

\begin{abstract}
Human system is made up of many organs; of all brain is the first and the leading controller of the human system. Overload cells growing in an uncontrolled manner in brain is called as brain tumor which further leads to brain cancer. MRI(Magnetic Resonance Imaging) is a medical test which uses strong magnets to produce magnetic field and radio waves to generate $2 / 3$ Dimensional image of different body organs and uses computer to analyze the taken image. The brain is composed of 3 types of materials: White Material (WM), Grey Matter (GM) and Cerebral Spinal Fluid (CSF).Through the MRI scan we can view the brain in three different ways: 1]The Axial MRI 2]The Sagittal MRI 3]The Coronal MRI. These images help the Doctor to identify whether that patient is suffering from cancer. The proposed system takes Brain MRI images as an input and pre-processing is performed on it (resizing and renaming). The images will be analyzed using advance imaging technologies. These technologies use Convolution Neural Network and deep learning approach for analysis. After analysis, classifying of whether given MRI images are normal or show a benign or malignant cancer is done automatically, that saves the radiologist's time, increases accuracy and yield of diagnosis.
\end{abstract}

Keywords: MRI Images, U-Net, Ground Truth, Tensor Flow, Tumour, Brain, Processing, classification, Benign, Malignant

\section{INTRODUCTION}

Brain cancer detection is a major technical challenge in medical diagnosis system So, This paper implement the detection of brain cancer. User can take MRI image as input and give it to system. The MRI scanner captures this energy and creates a picture of the tissues scanned based on this information. Then using CNN algorithm they perform the some operations like, Pre-processing ,Analysis, Conversion, Classification. using Deep learning technology on the MRI images and the result will be classification of brain MRI to detect the image depicts brain cancer Our automated system will help to analyze the brain MRI Image.

\section{LITERATURE SURVEY}

"Detection of Tumor in MRI Images Using Artificial Neural Networks" Aqhsa Q. Syed, K. NarayananInternational Journal of Engineering Research \& Technology (IJERT) September 2014.

Automatic defects detection in MR images is very important in many diagnostic and therapeutic applications. This work has introduced one automatic brain tumour detection method to increase the accuracy and yield and decrease the diagnosis time. The goal is classifying the tissues to two classes of normal and abnormal. MR images that have been used here are MR images from normal and abnormal brain tissues. This method uses from neural network to do this classification. The purpose of this project is to classify the brain tissues to normal and abnormal classes automatically, that saves the radiologist time, increases accuracy and yield of diagnosis.

“Classification of Brain Cancer Detection by using Magnetic Resonance Imaging” Vishal S. Shirsat, Seema S. Kawathekar--International Journal of Engineering Research \& Technology (IJERT) Vol. 3 Issue 2, Feb - 2014. The brain tumor is abnormal growth of cells inside skull which causes damage of the other cells necessary for functioning human brain. The brain tumor detection is challenging task due to complex structure of human brain. MRI images generated from MRI scanners using strong magnetic fields and radio waves to form images of the body which helps for medical diagnosis. This paper gives the overview of the various techniques used to detect the tumor in human brain using MRI images.

E.Lashkari, “A Neural Network- Based Method for Brain Abnormality Detection in MR Images Using Zernike Moments and Geometric Moments", International Journal of Computer Applications,Vol. 4, Issue.7, pp. 1-8, 2010.

Nowadays, automatic defects detection in MR images is very important in many diagnostic and therapeutic applications. This paper introduces a Novel automatic brain tumor detection method that uses T1, T2_weighted and PD, MR images to determine any abnormality in brain tissues. Here, has been tried to give clear description from brain 
tissues using Gabor wavelets, energy, entropy, contrast and some other statistic features such as mean, median, variance, correlation, values of maximum and minimum intensity .It is used from a feature selection method to reduce the feature space too. This method uses from neural network to do this classification. The purpose of this project is to classify the brain tissues to normal and abnormal classes automatically, that saves the radiologist time, increases accuracy and yield of diagnosis.

\section{PROPOSE SYSTEM}

In Proposed system we are analysing detection of brain cancer .User can take the MRI image and give it to automated system. Then system Using CNN algorithm can find the brain cancer for particular brain part. Then using Algorithm User input image as a MRI image is give to automated system and they perform the pre-processing on that MRI image. Then it will perform the second operation such as a conversion .in that the some type of conversion is Grayscale detection, Colour shape,etc.then this conversion output is input of analysis. Then image is analysis and it generate the result to user.

\section{SYSTEM ARCHITECTURE}

Following diagram is our system's architecture diagram:

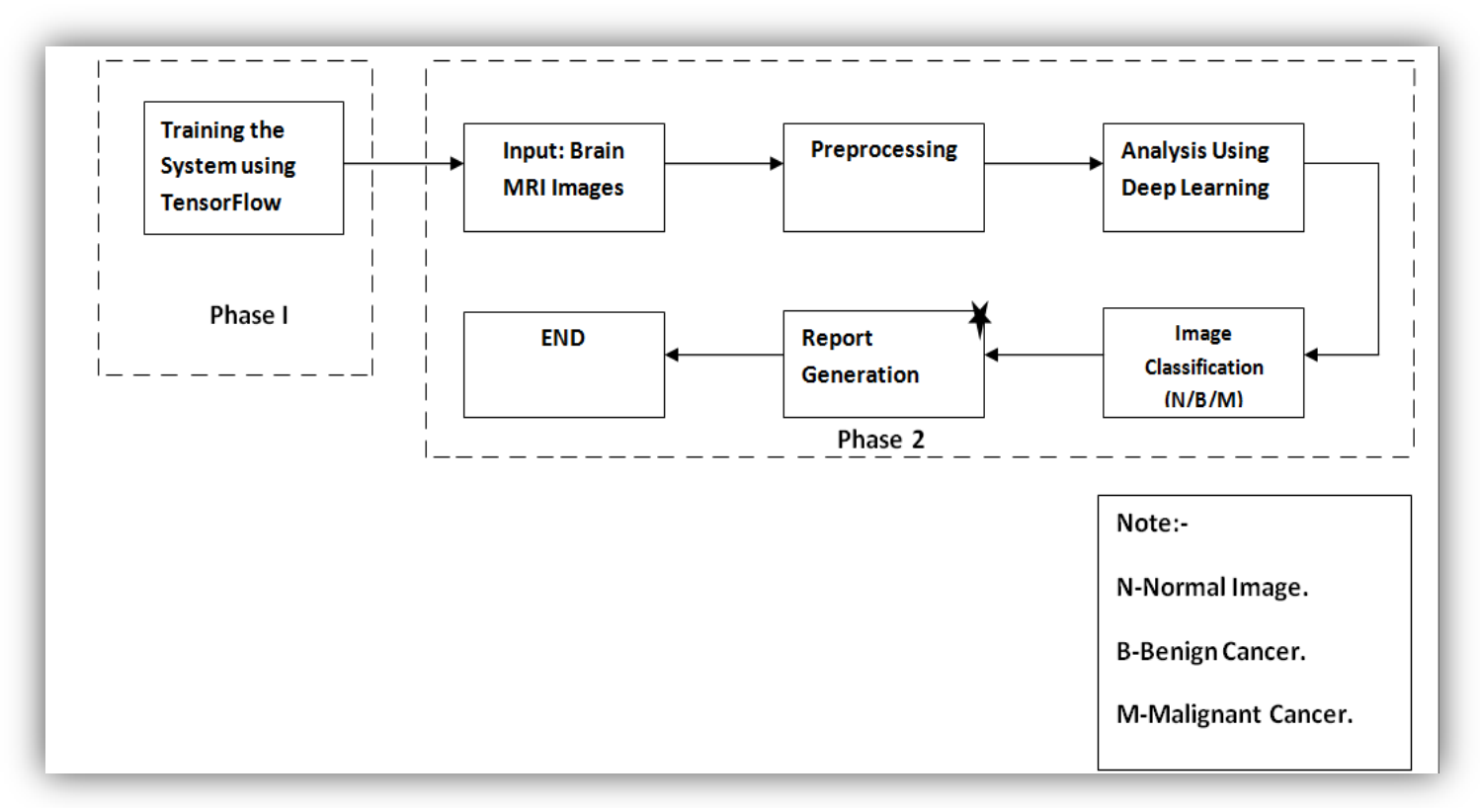

Figure 1: system architecture

Training data have a MRI image as a input.so this image can be pre-processing and pre-processing is done then it will move to Analysis phase. Analysis is using deep learning it perform the operation and it move to next phase that is Image Classification .classification perform the operation and it will generate the result.

\section{METHODOLOGIES}

Some methods are used for detection of brain cancer :-

1.Pre-Processing :- In that the image are resize or renamed.

2.Analysis using deep learning :-In that processed images are analyzed using deep learning technology.

3.Classification :- After analyses the images are classified according to the presence of cell.

\section{CONCLUSION}

In this proposed work different medical images like, MRI brain cancer images are taken for detecting Tumor. The proposed approach for Brain Tumor Detection based on Convolution Neural Network categorizes into Multi-layer Perceptron Neural Network. The proposed approach utilizes a combination of this neural network technique and is composed of several step including:-Training the system, Pre-Processing, Implementation of the Deep Learning, Classification. In future we will take a large database and try to give more accuracy which will work on any type of MRI Brain Tumor. 
International Journal of Advanced Research in Computer and Communication Engineering

Vol. 7, Issue 11, November 2018

\section{REFERRENCES}

[1]. Classification of Brain Cancer Detection by using Magnetic Resonance Imaging-Vishal S. Shirsat, Seema S. Kawathekar--International Journal of Engineering Research \& Technology (IJERT) Vol. 3 Issue 2, February - 2014

[2]. Detection of Tumor in MRI Images Using Artificial Neural Networks-Aqhsa Q. Syed, K. Narayanan- International Journal of Engineering Research \& Technology (IJERT) September 2014.

[3]. http://www.webmd.com/brain/magneticresonance- imaging-mri-of-the-head

[4]. E.Lashkari, "A Neural Network- Based Method for Brain Abnormality Detection in MR Images Using Zernike Moments and Geometric Moments”, International Journal of Computer Applications, Vol. 4, Issue.7, pp. 1-8, 2010

[5]. https://googleweblight.com/i?u=http://machinelearningmastery.com/whatisdeeplearning/\& grqid=OLXTugJr\&hl=en IN\&geid=1007. 